\title{
Angioleiomyoma masquerading as shoulder pain
}

\author{
Surekh Ravi ${ }^{1}$, Lazar J Chandy ${ }^{1}$, Gautam Kumar ${ }^{1}$, Biju Jacob ${ }^{1}$, Ami M Emmanuel ${ }^{2}$ \\ ${ }^{1}$ Department of Orthopedics, VPS Lakeshore Hospital, Kochi, India \\ ${ }^{2}$ Department of Pathology, VPS Lakeshore Hospital, Kochi, India
}

\begin{abstract}
Angioleiomyoma is a benign soft tissue tumor originating from vascular smooth muscle. We report a case of a 20 -year-old student who presented with pain in the right shoulder of 4 years duration. Shoulder movements were pain-free throughout the range of motion except resisted external rotation. Magnetic resonance imaging visualized a well-circumscribed lesion over the infraspinatus tendon. The lesion was surgically removed and sent for histopathological analysis. Morphology and immunohistochemistry results were suggestive of angioleiomyoma. The most common location for such a lesion is the lower limb, with less than $1 \%$ being reported in the upper arm, of which an angioleiomyoma of the shoulder is extremely rare.
\end{abstract}

Keywords: Leiomyoma; Magnetic resonance imaging; Soft tissue neoplasms

Angioleiomyoma is a benign soft tissue tumor that originates from vascular smooth muscle. These are well-circumscribed tumors usually round or oval in shape, commonly presenting as a painful mass. Microscopically, the lesion consists of numerous fine blood vessels of varying size surrounded by a smooth muscle bundle. The World Health Organization has classified them as benign perivascular tumors [1]. The tumor usually presents in the lower extremities, with less than 1\% [2] being described in the upper limb. We report a case of a 20 -year-old male who had symptomatic angioleiomyoma in the right shoulder.

\section{CASE REPORT}

This study was approved by the Institutional Review Board of CDSCO India, Lakeshore Ethics Committee (No. ECR/120/ LAKESHORE/INST/KER/2013/RR-16). The informed consent was received from the patient for publication.
A 20-year-old male visited our out patient department complaining of pain in the right shoulder of 4-year duration. He was a right-handed individual with no comorbidities. The pain was insidious in onset, gradual in progression, absent upon rest, with no diurnal variation, and aggravated by strenuous exercise such as swimming and weightlifting. The visual analog scale score was $6 / 10$ at presentation. The patient had received one intra-articular injection at another clinic suspecting supraspinatus tendinitis, which offered transient pain relief for 1 month.

The clinical examination revealed no deformity, visible swelling, or wasting of muscles. The patient had full pain-free range of movement. The pain was elicited upon resisted external rotation. The laboratory investigations, including total leukocyte count and erythrocyte sedimentation rate, were within normal limits excluding the infective pathology. The plain radiograph of the shoulder was within normal limits. A Siemens Magneton Skyra 3 Tesla (Siemens, Erlangen, Germany) magnetic resonance imag-

Received: July 22, $2020 \quad$ Revised: August 28, $2020 \quad$ Accepted: August 29, 2020

Correspondence to: Lazar J Chandy

Department of Orthopedics, VPS Lakeshore Hospital, Kochi, Kerala 682040, India

Tel: +91-9447391770, E-mail: lazarjchandy@gmail.com, ORCID: https://orcid.org/0000-0003-4521-550X

\section{Financial support: None.}

Conflict of interest: None.

Copyright@ 2021 Korean Shoulder and Elbow Society. All Rights Reserved.

This is an Open Access article distributed under the terms of the Creative Commons Attribution Non-Commercial License (http://creativecommons.org/licenses/by-nc/4.0/) which permits unrestricted non-commercial use, distribution, and reproduction in any medium, provided the original work is properly cited. 
ing (MRI) instrument was used to visualize a $1.3 \times 1.2 \times 1.4 \mathrm{~cm}$, well-circumscribed lesion that was isointense in T1-weighted (T1W) and hyperintense on T2-weighted (T2W) images, along the posterior superficial surface of the infraspinatus tendon (Figs. 1 and 2) near its attachment.

The provisional diagnosis of giant cell tumor (GCT) of the tendon sheath was based on the peritendinous location and T2 hyper-intensity on contrast-enhanced imaging. A ganglion cyst was also considered as a provisional diagnosis due to the well-circumscribed margins and T2 hyper-intensity of the lesion. Ultrasound-guided fine needle aspiration was planned but omitted in view of the high risk of field contamination in suspected malignancy. Open excision biopsy was preferred over arthroscopic biopsy as we considered a differential diagnosis of GCT and possibility of malignant tumor. To avoid tissue contamination and

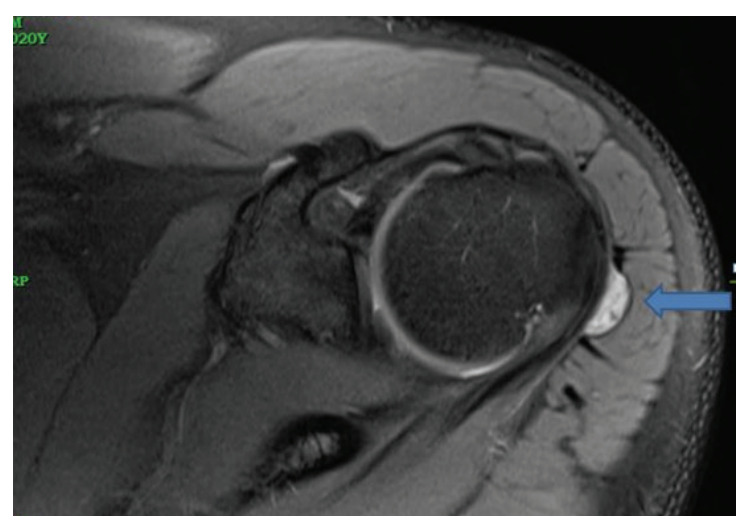

Fig. 1. An axial proton density fat saturated image showing a well-demarcated hyperintense lesion along the posterior surface of the infraspinatus tendon protruding into the subacromial subdeltoid bursa. The single arrow in axial proton density fat saturated image showing well demarcated hyperintense lesion along the posterior surface of infraspinatus tendon protruding into the subacromial subdeltoid bursa.

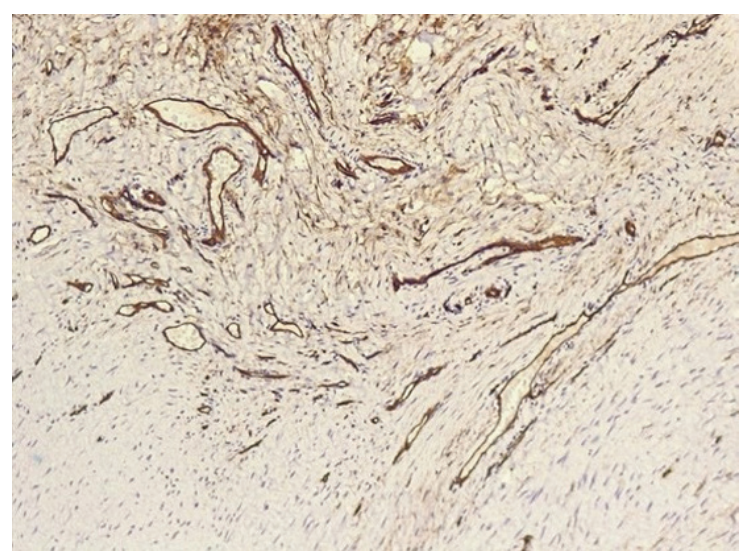

Fig. 2. A coronal T2 fat suppressed image showing a well circumscribed hyperintense lesion along the superficial surface of the infraspinatus tendon protruding into the subacromial subdeltoid bursa. complete removal, we performed open marginal excision of the lesion.

The patient was placed in the beach chair position under general anesthesia, and the lesion was visualized using a posterior approach through a horizontal incision after retracting the deltoid fibers. A well-encapsulated lobulated mass was found lying over the infraspinatus muscle in close proximity but not adherent to the axillary nerve. The mass was removed intact along with part of the subacromial bursa, and the tissue was sent for histopathological evaluation. On gross examination (Fig. 3), a circumscribed firm nodule was observed with adjacent skeletal muscles. The microscopic examination was suggestive of a well-circumscribed neoplasm that was mainly composed of spindly cells; devoid of significant atypia or mitotic activity. The delicate and branching vessels (Fig. 4) were observed within the lesion. On immunohistochemistry (IHC) examination, the spindly cells were positive for vimentin, smooth muscle actin (SMA) (Fig. 5), and caldesmon. The prominent vasculature within the lesion was

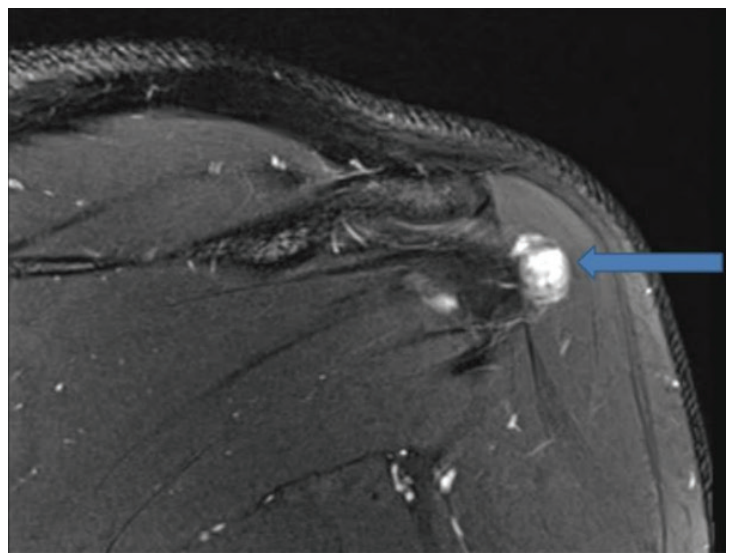

Fig. 3. Excised gross specimen showing a globular mass with skeletal muscle attached.

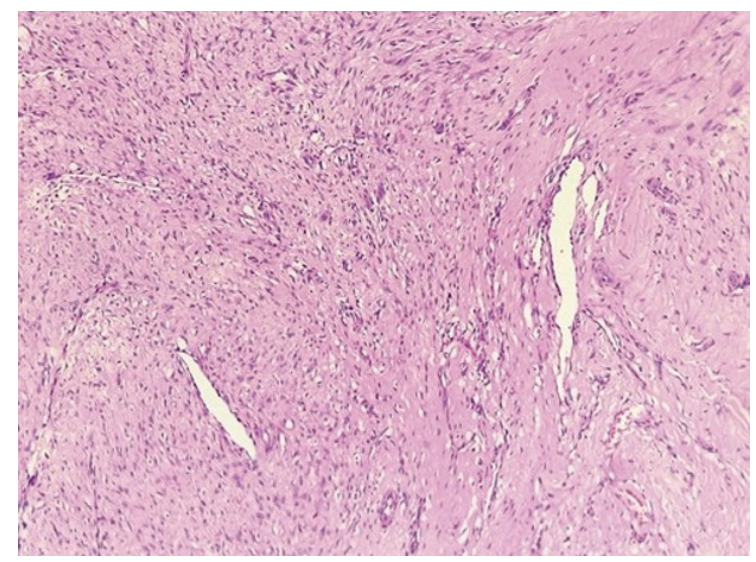

Fig. 4. H\&E-stained $40 \times$ magnification showing bland spindle cells with interspersed vascular channels. 


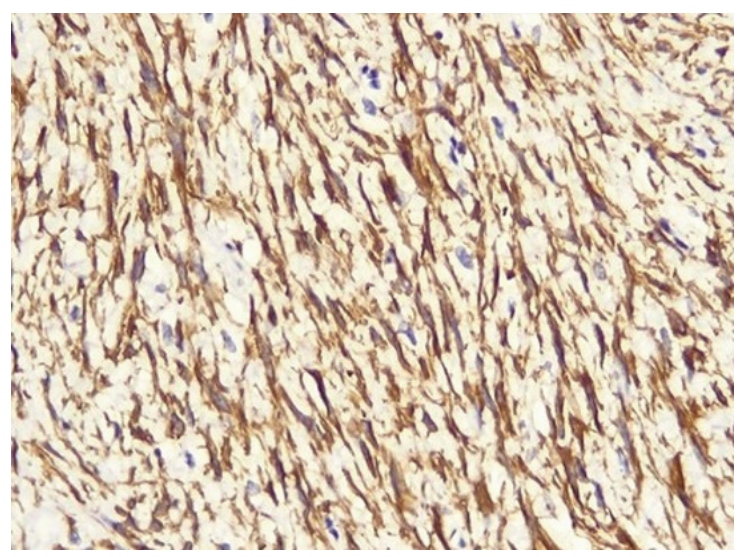

Fig. 5. Immunohistochemistry smooth muscle actin (SMA) staining at $400 \times$ showing cytoplasmic positivity in the spindly cells.

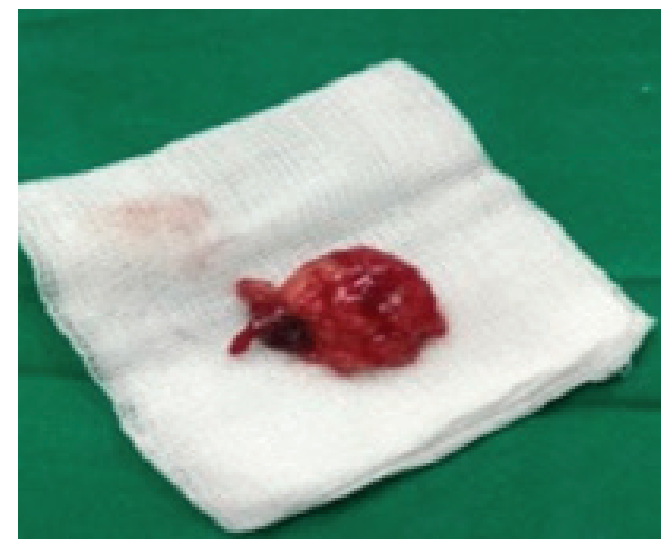

Fig. 6. Image of $400 \times$ magnification of immunohistochemistry CD34 showing cytoplasmic and membranous positivity in lining endothelial cells.

highlighted by CD34 (Fig. 6).

The morphology and IHC results were suggestive of angioleiomyoma. The patient was prescribed passive mobilization of shoulder from post-operative day 1 . The patient was evaluated at 2 and 6 months post-surgery. Shoulder movements were painfree throughout the range of motion, including resisted external rotation.

\section{DISCUSSION}

Angioleiomyomas are benign soft tissue tumors first described by Stout [3]. Such tumors originate from vascular smooth muscle (tunica media) [4] and comprise around 5\% of all soft tissue tumors, of which tumors involving the upper arm represent $<1 \%$ [2]. Angioleiomyomas usually present at 40-60 years of age, in female patients. The lower extremities are involved in more than $2 / 3$ of cases [2]. A benign nodule is $<2 \mathrm{~cm}$ in size [5] with pain as the presenting symptom in around $50 \%$ of cases [5]. These are well-circumscribed encapsulated tumors usually round or oval in shape. Microscopically, the lesion consists of numerous fine blood vessels of varying size surrounded by smooth muscle bundle [6]. The characteristic spindle-shaped cells have elongated cigar-like nuclei and eosinophilic cytoplasm. IHC results of spindle cells will be positive for SMA and vimentin, while endothelial cells in the vessel will stain positive for CD 34. It is postulated that the presence of neural elements is responsible for patient pain [7]. Transient ischemia due to contraction of smooth muscles is another theory postulated for pain source [2].

The differential diagnosis includes GCT of the tendon sheath and ganglionic cyst. Morimoto [8] classified angioleiomyoma into three types: solid, venous, and cavernous based on histopathology. The case discussed above was a solid variant of angioleiomyoma. MRI characteristics of angioleiomyoma have been well described. The lesion appears isointense to skeletal muscle on T1- and hyperintense on T2W images. The hyperintense area in the $\mathrm{T} 2 \mathrm{~W}$ image corresponds to the smooth muscle component [9] of the lesion. In angioleiomyoma, MRI lacks specificity in the absence of any specific radiological features. Complete excision of angioleiomyoma is the treatment of choice, as it is both diagnostic as well as therapeutic. The diagnosis is confirmed with histopathological analysis of the excision biopsy sample, supported by IHC analysis. Potential complications include recurrence after excision and malignant transformation to angioleiomyosarcoma. Malignant transformation of angioleiomyoma to angioleiomyosarcoma is extremely rare, with only a few cases [10] described.

Angioleiomyoma is a rare benign soft tissue tumor arising from tunica media of blood vessels. The most common location for the lesion is the lower limb, with less than $1 \%$ being reported in the upper arm, of which angioleiomyoma of the shoulder is an extremely rare tumor. when dealing with a benign swelling over the shoulder joint, angioleiomyoma should be kept in the list of differential diagnosis. The diagnosis is made by excision biopsy followed by histopathological evaluation.

\section{ACKNOWLEDGMENTS}

We would like to acknowledge Dr. Pushpa Mahadevan, Department of pathology, VPS Lakeshore Hospital, Kochi, Kerala and Dr. Shruti P, Department of pathology, ASTER MIMS, Kanoor, Kerala for valuable feedback in histopathological analysis and reporting of the tumor and Dr. Julio C Kandathil for radiological analysis of the tumor. 


\section{ORCID}
Surekh Ravi
https://orcid.org/0000-0001-9251-978X
Lazar J Chandy
https://orcid.org/0000-0003-4521-550X
Gautam Kumar
Biju Jacob
https://orcid.org/0000-0001-9307-6159
https://orcid.org/0000-0001-8491-4755
Ami M Emmanuel

\section{REFERENCES}

1. Fletcher CD. The evolving classification of soft tissue tumoursan update based on the new 2013 WHO classification. Histopathology 2014;64:2-11.

2. Hachisuga T, Hashimoto H, Enjoji M. Angioleiomyoma. A clinicopathologic reappraisal of 562 cases. Cancer 1984;54:126-30.

3. Stout AP. Solitary cutaneous and subcutaneous leiomyoma. Am J Cancer 1937;29:435-69.

4. Cherrick HM, Dunlap CL, King OH Jr. Leiomyomas of the oral

cavity: review of the literature and clinicopathologic study of seven new cases. Oral Surg Oral Med Oral Pathol 1973;35:5466.

5. Duchateau J, Zielonka E, Guelinckx PJ. Chronic pain: illusion or pathology? A case report of a vascular leiomyoma in the leg. Br J Plast Surg 1987;40:536-7.

6. Dicaprio MR, Jokl P. Vascular leiomyoma presenting as medial joint line pain of the knee. Arthroscopy 2003;19:E24.

7. Montgomery H, Winkelmann RK. Smooth-muscle tumors of the skin. AMA Arch Derm 1959;79:32-40.

8. Morimoto N. Angiomyoma (vascular leiomyoma): a clinicopathologic study. Med J Kagoshima Univ 1973;24:663-83.

9. Ramesh P, Annapureddy SR, Khan F, Sutaria PD. Angioleiomyoma: a clinical, pathological and radiological review. Int J Clin Pract 2004;58:587-91.

10. Herren DB, Zimmermann A, Büchler U. Vascular leiomyoma in an index finger undergoing malignant transformation. J Hand Surg Br 1995;20:484-7. 